\title{
Metastable Switching in a Planar Limit Cycle System with
}

\section{Additive Noise}

\author{
Michael A. Schwemmer* and Jay M. Newby \\ Mathematical Biosciences Institute, The Ohio State University, 1735 Neil Ave. Columbus, OH 43210
}

\begin{abstract}
Additive noise is known to produce counter-intuitive behaviors in nonlinear dynamical systems. Previously, it was shown that systems with a deterministic limit cycle can display switching between metastable states in the presence of asymmetric additive white noise. Here, we systematically analyze the dynamics of this metastable behavior and show how the vector field away from the limit cycle influences the rate and directionality of the metastable switching. Using stochastic phase reduction methods as well as asymptotic approximations, we identify mechanisms underlying different rates of switching and predict when the system will rotate in the opposite direction of the deterministic limit cycle. Thus, this work presents an alternative mechanism for generating a range of metastable switch behaviors that have been observed in a number of physical systems.
\end{abstract}

Key words: stochastic differential equations; limit cycle oscillator; phase reduction; metastable switching

\section{Introduction}

Deterministic limit cycle oscillators are used to model nonlinear phenomena observed throughout nature $[14,21,30]$. However, in certain cases (e.g. neural oscillators and cell cycle reactions), stochastic fluctuations are observed in the oscillatory dynamics, motivating a recent push to develop a theoretical understanding of how noise affects limit cycle oscillators. [11,12,28,31].

Simple additive white noise can significantly alter the dynamics of nonlinear systems. For example, it has been shown that additive white noise can make a nonlinear excitable systems display coherent limitcycle-like oscillations $[5,17,22]$. In previous work [20], we showed that a system with a deterministic limit cycle subject to additive white noise can display numerous interesting and counter-intuitive behaviors. In some cases, the addition of noise can act to completely eliminate oscillations, and even cause the trajectories to rotate in the opposite direction of the deterministic limit cycle. Perhaps more interesting is the emergence of metastable switching behavior similar to random motion in a tilted periodic potential, where the potential has one or more wells within each period that confine the motion.

Metastable switching behavior has been observed in variety of physical systems, including gene networks $[10,15]$, neural systems [4,8], and neural growth processes [2]. In the simplest example, metastable switching arises when noise is added to a system that has deterministic bistability, i.e., Kramers' problem [9]. In this case, the stochastic process will randomly switch between the two deterministic fixed points (left panel in Figure 1). The resulting stationary density of the process will have two distinct peaks centered around the deterministic stable fixed points (right panel in Figure 1). In [20], we demonstrated that similar switching dynamics arise when noise is added to systems with a deterministic limit cycle. Importantly, in the main examples studied in [20], the limit cycle always exists, and thus the

*Corresponding Author: schwemmer.2@mbi.osu.edu 


\section{${ }_{76} 2$ Models} ways. in the phase variable.

points where the stochastic process spent most of its time represent metastable points that do not exist in the deterministic system. Through the analysis of two simple planar limit cycle systems, we were able to identify precisely when this metastable switching will occur. Furthermore, we were also able to numerically demonstrate that this type of metastable switching can occur in the more complex FitzHughNagumo [7,18] oscillator model ( [20]; cf. Fig. 6) . However, metastability in these cases was diagnosed only using the stationary density, which ignores the dynamics of the switching process. Indeed, the underlying dynamics of the metastable behavior in these systems can be quite variable (see Figure 3). As such, we now systematically analyze the dynamics of this metastable switching behavior in these simple systems, and identify the mechanisms responsible for different types of switching dynamics.

The interaction of the additive noise with the dynamics away from the deterministic limit cycle is the key to understanding the different switching behaviors. The two planar limit cycle systems we analyze have areas of the vector field where the rotation occurs in the opposite direction of the limit cycle, and each system differs in the placement of these areas. In one system, which we refer to as counterrotating $(\mathrm{CR})$, the rotation changes direction either inside the limit cycle or outside the limit cycle, depending on parameter values. In the other system, which we refer to as antirotating (AR), the rotation changes direction on both sides of the limit cycle. We will show that these different vector fields lead to very different metastable switching behaviors when perturbed by white noise.

We apply the same additive noise to the $y$ coordinate of each system. Due to the nonlinear interactions with the deterministic dynamics, the fluctuations in the angular phase coordinate (the direction tangent to the limit cycle) is very different for each system. In the counterrotating system, the dynamics amplifies noise in the phase coordinate, while the antirotating system suppresses it. This is important for understanding the metastable switching behavior because the metastable transitions take longer to occur when the noise is suppressed. Through the use of stochastic phase reduction methods [28, 31], we show that the directionality of switching is controlled by the drift of the phase reduced system while the rate of switching, similar to the Kramers' problem, is determined by the magnitude of the fluctuations in phase. To accomplish this, we derive asymptotic approximations to the stationary densities of the phase reduced systems. We also use numerical simulations to verify our results, and to identify regions in parameter space where the approximations break down. Importantly, this work illustrates that a wide range of metastable switching dynamics can be modelled by limit cycle systems with additive noise, and provides further evidence that noise can affect seemingly similar nonlinear systems in strikingly different

The paper is organized as follows. First we present the equations for the two planar limit cycle systems we analyze. Next, we give the details of the transformation of the system to asymptotic phase which then allows us to reduce the two dimensional systems to a single stochastic differential equation for the evolution of the phase variable. We then review how the onset of metastable switching behavior can be predicted from the phase model by examining the drift term in the conservation (Fickian) form of the stationary Fokker-Planck equation. We then illustrate that even though the two systems display qualitatively similar stationary densities, the dynamics of the metastable switching in each system is quite different. By deriving asymptotic approximations to the stationary flux, we show that the directionality of the switching is determined by the sign of the flux. Lastly, by exploring the terms in the reduced phase system, we show that the rate of switching is determined by the magnitude of the stochastic fluctuations

To explore this metastable switching behavior in limit cycle sytems, we consider the following analytically tractable deterministic oscillator

$$
\begin{aligned}
& \dot{x}=-\omega y+\gamma x\left(1-\rho^{2}\right)+c \gamma y Q(\rho) \equiv F(x, y) \\
& \dot{y}=\omega x+\gamma y\left(1-\rho^{2}\right)-c \gamma x Q(\rho) \equiv G(x, y)
\end{aligned}
$$


where $\rho=\sqrt{x^{2}+y^{2}}$ and the function $Q(\rho)$ is such that $Q(1)=0$ and determines the rotation away from the limit cycle. The dynamics of the system are easier to understand if (2.1) is rewritten in polar coordinates

$$
\begin{aligned}
& \dot{\theta}=\omega-\gamma c Q(\rho) \\
& \dot{\rho}=-\gamma \rho\left(\rho^{2}-1\right) .
\end{aligned}
$$

In particular, it can now easily be seen how $Q(\rho)$ affects the rotation away from the limit cycle at $\rho=1$. We assume that the limit cycle is strongly attracting so that $\gamma \gg \omega$ is a large parameter. If $c=0$, then (2.2) is independent of $Q$ and yields the radial isochron clock model when $\gamma=1$. In this case, the rotation $\dot{\theta}$ is constant away from the limit cycle and independent of $\rho$. If $c \neq 0$, then the rotation changes direction for values of $\rho=\rho_{*}$ such that $Q\left(\rho_{*}\right)=\omega /(c \gamma)$. We consider the following two cases:

$$
\begin{aligned}
& Q_{1}(\rho)=\rho^{2}-1 \\
& Q_{2}(\rho)=\omega(1-\rho)^{2} .
\end{aligned}
$$

$Q_{1}$ yields the Stuart-Landau model [26], which has been used to model the shedding of vortices in the two-dimensional wake of a cylinder at low Reynolds number (e.g., [16,24]). However, for reasons made clear below, we will refer to the system with $Q_{1}$ as the counterrotating (CR) whereas we will refer to $Q_{2}$ as the antirotating (AR) model. It follows that the limit cycle rotates counter clockwise with period $2 \pi / \omega$ on $\rho=1$.

Figure 2 plots the vector field of (2.1) for the two different choices of the function $Q(\rho)(2.3)$. Note that, regardless of the definition of the function $Q(\rho)$, the limit cycle is given by $x(t)=\cos (\omega t)$ and $y(t)=\sin (\omega t)$ (thick black line). For the CR system, there is a unique value of $\rho_{*}=\sqrt{1+\omega /(c \gamma)}$ where the rotation changes sign (dashed line); if $c>0$ then $\rho_{*}>1$ and if $c<0$ then $\rho_{*}<1$ (not shown). On the other hand, for the AR system, we have that $\rho_{*}=1 \pm \sqrt{1 /(c \gamma)}$. Hence, there are two values of $\rho_{*}$ if $c>0$ (one inside the limit cycle and one outside, dashed lines) and none if $c<0$. For simplicity, we only consider the $c>0$ case for both systems. Note that for both the CR and AR systems, increases in $c$ and $\gamma$ cause $\rho^{*}$ to move closer to the limit cycle.

Next, we add independent white noise to each component

$$
\begin{aligned}
& \dot{x}=F(x, y)+\sigma w_{x} \xi_{x}(t) \\
& \dot{y}=G(x, y)+\sigma w_{y} \xi_{y}(t)
\end{aligned}
$$

where $\sigma$ is the noise strength, $w_{x}$ and $w_{y}$ are constants, and $\xi_{i}(t), i=x, y$, is Gaussian white noise with zero mean and unit variance (i.e., $\left\langle\xi_{i}(t)\right\rangle=0$ and $\left\langle\xi_{i}(t) \xi_{i}(s)\right\rangle=\delta(t-s)$ ). If $w_{x}=w_{y}$, then the stochastic process is rotationally symmetric. In this case, the CR system acts to amplify noise fluctuations whereas in the AR system, depending on the value of $c$, the noise can cause the system to rotate in the opposite direction of the deterministic limit cycle or even cease oscillating altogether [20]. When the statistical rotational symmetry is broken, $w_{x} \neq w_{y}$, it was also shown that the CR and AR systems can display metastable switching between two metastable states ( [20] and see below). Since we are interested in further examining this metastable behavior, for the rest of the paper we assume, without loss of generality, that $w_{x}=0$ and $w_{y}=1$. This simplifies (2.4) to the following system of stochastic differential equations (SDEs)

$$
\begin{aligned}
& \dot{x}=F(x, y) \\
& \dot{y}=G(x, y)+\sigma \xi(t),
\end{aligned}
$$

To simulate the SDEs, we employ the Euler-Mayurama method with a sufficiently small time step. 


\section{Metastable Switching in Limit Cycle Systems}

In previous work, it was shown that with $c \approx 1$ and moderate levels of noise $\sigma$, the two planar limit cycle systems exhibit bistable switching. Figure 3 plots example trajectories for both systems. For the CR system, the $x$ coordinate appears to display strong metastable switching between \pm 1 at a fairly frequent rate. However, for the AR system, it is the $y$ coordinate that appears most like a metastable switch, but does so at a rate much slower than that of the CR system. This shows that the vector field away from the limit cycle interacts with the noise in interesting ways in order to produce this behavior. Thus, we seek to understand (i) how this metastable behavior emerges in each of these systems, and (ii) why metastable switching is different between the two systems. To do so, we first utilize a phase reduction technique for stochastic limit cycle systems $[28,31]$ in order to reduce the system to a one-dimensional Ito SDE which describes the position of the system on its underlying determinsitic limit cycle.

\subsection{Reduction to a Phase Model}

In the absence of noise both planar sytems (2.1) exhibit stable limit cycle oscillations. Since we also assume that the rate of attraction back to the limit cycle is large, i.e., $\gamma \gg \omega$, this places us in the ideal situation to use so-called phase reduction techniques $[6,14,28,31]$. In this case, the dynamics of a single phase variable $\varphi$ that denotes the location of a trajectory on the underlying limit cycle will be an accurate (to order $1 / \gamma$ ) description of the full dynamics of the system.

Consider the noiseless system $(2.1)$ which has a stable $2 \pi / \omega$-periodic solution. We define a phase $\varphi \in[0,2 \pi / \omega)$ coordinate on the limit cycle solution such that it increases by $2 \pi / \omega$ for every cycle of the system. That is, $\varphi$ is defined by

$$
\frac{d \varphi}{d t}=\frac{\partial \varphi}{\partial x} \frac{d x}{d t}+\frac{\partial \varphi}{\partial y} \frac{d y}{d t} \equiv 1 .
$$

We use phase instead of polar coordinates because, in the absence of external input, the phase variable $\varphi$ increases monotonically with a constant rate. Thus, any change to the rate of increase of the phase variable will solely be due to the external input (in this case noise) and not due to the intrinsic dynamics of the system. This is not the case for the angle variable $\theta$ in polar coordinates (2.2).

In general, it is not possible to find the exact mapping from Cartesian coordinates to $\varphi$. However, for the planar systems we use here, the mapping $(x, y) \mapsto \varphi$ can be found analytically. To do so, we first transform the partial differential equation in (3.1) to polar coordinates

$$
\begin{aligned}
1 & =\frac{\partial \varphi}{\partial \theta} \frac{d \theta}{d t}+\frac{\partial \varphi}{\partial \rho} \frac{d \rho}{d t} \\
& =\frac{\partial \varphi}{\partial \theta}(\omega-\gamma c Q(\rho))+\frac{\partial \varphi}{\partial \rho}\left(-\gamma \rho\left(\rho^{2}-1\right)\right) .
\end{aligned}
$$

The coefficients in the above equation only depend on $\rho$, so we can try a solution of the form $\varphi(\theta, r)=$ $[\theta+\Lambda(\rho)] / \omega$. Substitution leads to

$$
1=\frac{1}{\omega}\left[\omega-c \gamma Q(\rho)-\gamma \rho\left(\rho^{2}-1\right) \Lambda^{\prime}(\rho)\right],
$$

which implies that

$$
\Lambda(\rho)=-c \int_{1}^{\rho} \frac{Q(\tilde{\rho})}{\tilde{\rho}\left(\tilde{\rho}^{2}-1\right)} d \tilde{\rho} .
$$

Next, we define a new amplitude variable $r=\rho-1$ which is the distance of the system from the deterministic limit cycle. Using the fact that $\theta=\tan ^{-1}(y / x)$ and $\rho=\sqrt{x^{2}+y^{2}}$, we arrive at our desired mapping 


$$
\varphi=\frac{1}{\omega}\left[\tan ^{-1}(y / x)+c H(r)\right], \quad r=\sqrt{x^{2}+y^{2}}-1,
$$

where $H(r) \equiv \frac{1}{c} \Lambda(r+1)$. It is important to note that the function $H(\cdot)$ depends on the function $Q(\cdot)$. Thus, the mapping to phase will be different for the CR and AR systems.

Although phase is defined on the limit cycle, it is extended to points off the limit through the use of asymptotic phase, or isochrons [30]. That is, isochrons are lines of constant $\varphi$, and all deterministic trajectories starting on an isochron converge to the same point on the limit cycle as $t \rightarrow \infty$. Thus, the phase mapping (3.3) takes points off the limit cycle and maps them to their asymptotic phase value on the limit cycle. Isochrons also encode information about how the phase of a trajectory responds to a perturbation away from the limit cycle. Indeed, the gradient of this mapping $\frac{\partial \varphi}{\partial i}, i=x, y$ is typically referred to as the phase response curve of the $i$-th component of the limit cycle $[6,14,25]$ as it quantifies the change in phase due to a $\delta$-function perturbation at a particular phase on the limit cycle.

With our mapping in hand (3.3), we transform the stochastic Cartesian system $(2.5)$ to $(\varphi, r)$ coordinates

$$
\begin{aligned}
\dot{\varphi} & =1+\frac{\sigma^{2}}{2} n(\varphi, r)+\sigma h(\varphi, r) \xi(t) \\
\dot{r} & =\gamma f(r)+\frac{\sigma^{2}}{2} n_{r}(\varphi, r)+\sigma g(\varphi, r) \xi(t),
\end{aligned}
$$

where,

$$
\begin{aligned}
& h(\varphi, r) \equiv \frac{\partial \varphi}{\partial y}=\frac{1}{\omega}\left[\frac{\cos (\alpha)}{r+1}+c H^{\prime}(r) \sin (\alpha)\right], \quad g(\varphi, r) \equiv \frac{\partial r}{\partial y}=\sin (\alpha), \\
& f(r) \equiv \frac{\partial r}{\partial x} F(x(\varphi, r), y(\varphi, r))+\frac{\partial r}{\partial y} G(x(\varphi, r), y(\varphi, r))=-r(r+1)(r+2), \\
& \alpha \equiv \omega \varphi+c H(r),
\end{aligned}
$$

and we have used the inverse mappings $x(\varphi, r)=(r+1) \cos (\alpha)$ and $y(\varphi, r)=(r+1) \sin (\alpha)$. While the noise is additive in cartesian coordinates $(2.5)$, it is multiplicative in $(\varphi, r)$ coordinates. As such, we use the Ito interpretation. The terms that arise from the stochastic change of variables (i.e., the terms that come from the Ito calculus [9]) are $n(\varphi, r) \equiv h(\varphi, r) \frac{\partial h}{\partial \varphi}+g(\varphi, r) \frac{\partial h}{\partial r}$ and $n_{r}(\varphi, r) \equiv h(\varphi, r) \frac{\partial g}{\partial \varphi}+g(\varphi, r) \frac{\partial g}{\partial r}$. We stress that (3.4) is an exact transformation, and no approximations have been used up to this point. Thus, our goal now remains to project out the variable $r$ in order to arrive at a single differential equation for the phase.

Assuming that the deterministic limit cycle is highly attracting (i.e., $\gamma \gg \omega$ ), the system should cling tighly to the underlying deterministic limit cycle, and on average $r$ will be approximately 0 . In this case, it was previously shown $[28,31]$ that one can set $r=0$ in $(3.4)$ to obtain (to leading order in $\gamma^{-1}$ ) the one dimensional approximation

$$
\dot{\varphi} \sim 1+\frac{\sigma^{2}}{2} n_{0}(\varphi)+\sigma h_{0}(\varphi) \xi(t), \quad \gamma \gg \omega,
$$

where $n_{0}(\varphi) \equiv n(\varphi, 0)$ and $h_{0}(\varphi) \equiv h(\varphi, 0)$ is the phase response curve of the y-component of the deterministic limit cycle. Note that (3.5) can be rigorously derived by using adiabatic (or quasi-steady state) reduction techniques $[9,28]$.

The stationary density $u_{s s}(\varphi)$ of the reduced system (3.5) will provide insight into the dynamics of the full model. The stationary density obeys the following Fokker-Planck (FP) equation

$$
0=\partial_{\varphi}\left[\partial_{\varphi}\left\{D_{\varphi}(\varphi) u_{s s}\right\}-I_{\varphi}(\varphi) u_{s s}\right],
$$

with periodic boundary conditions. Note that in general analytical solutions to the above FP equation are not possible and we instead find approximate solutions using a forward or backward finite-difference 
scheme (depending on parameters). $I_{\varphi}(\varphi) \equiv 1+\frac{\sigma^{2}}{2} n_{0}(\varphi)$ is the Ito drift, or the deterministic portion of the $\operatorname{SDE}(3.5)$, and $D_{\varphi}(\varphi) \equiv \frac{\sigma^{2}}{2} h_{0}^{2}(\varphi)$ is the diffusivity. The FP equation (3.6) can also be written in conservation or Fickian form

$$
0=\partial_{\varphi}\left[D_{\varphi}(\varphi) \partial_{\varphi} u_{s s}-V_{\varphi}(\varphi) u_{s s}\right]
$$

where $V_{\varphi}(\varphi) \equiv I_{\varphi}(\varphi)-D_{\varphi}^{\prime}(\varphi)$ is the Fickian drift.

The Fickian drift is closely related to the stationary density of the the system. To see this, integrate both sides of (3.7) to obtain

$$
-J=D_{\varphi}(\varphi) \partial_{\varphi} u_{s s}-V_{\varphi}(\varphi) u_{s s},
$$

where $J$ is the probability flux. If the flux is zero, then $u_{s s}$ has the solution $u_{s s}(\varphi)=N e^{-\Phi(\varphi)}$ where

$$
\Phi(\varphi)=\int_{0}^{\varphi} \frac{-V(\tilde{\varphi})}{D(\tilde{\varphi})} d \tilde{\varphi}
$$

and $N$ is a normalization factor.

On the other hand, the Ito drift represents the average increment of the stochastic process during an infinitesimal time $d t$. For a periodic system such as ours, the Ito drift is also related to the stationary probability flux. To see this, integrate both sides of (3.6) to obtain

$$
-J=\partial_{\varphi}\left(D_{\varphi}(\varphi) u_{s s}\right)-I_{\varphi}(\varphi) u_{s s}
$$

where $J$ is again the probability flux. Lastly, integrating both sides of the above equation from 0 to $2 \pi / \omega$ (and noting that $D_{\varphi}(\varphi)$ and $u_{s s}$ are $2 \pi / \omega$ periodic) yields

$$
J=\frac{1}{2 \pi / \omega}\left\langle I_{\varphi}(\varphi)\right\rangle_{\varphi} \equiv \frac{1}{2 \pi / \omega} \int_{0}^{2 \pi / \omega} I_{\varphi}(\varphi) u_{s s}(\varphi) d \varphi .
$$

In the case that the stationary density is uniform $u_{s s}=(2 \pi / \omega)^{-1}$, the probability flux is proportional to the average Ito drift over one period of the oscillations. However, in general the stationary density will not be uniform, and its shape will have a large influence on the flux.

The flux can also be thought of as the average frequency of the oscillations. In the noiseless system, $I_{\varphi}(\varphi)=1$, and thus the flux is $(2 \pi / \omega)^{-1}$, which is the frequency of the deterministic limit cycle. In fact, for stochastically perturbed periodic systems that have an Ito drift of 1 , the stationary flux is always $(2 \pi / \omega)^{-1}$ regardless of the noise level.

\subsection{Diagnosing Metastability in the CR and AR Systems}

Before showing how the onset of metastable switching behavior can be predicted using the phase reduced system, we first give the details of the drift and diffusivity functions which will be vital to our later analysis. For the CR system, the Ito drift, Fickian drift, and diffusivity are given by

$$
\begin{aligned}
& I_{\varphi}^{C R}(\varphi)=1-\frac{\sigma^{2}}{2 \omega}[c \cos (2 \omega \varphi)+\sin (2 \omega \varphi)] \\
& V_{\varphi}^{C R}(\varphi)=1+\frac{\sigma^{2}}{2 \omega}\left[-c^{2} \sin (2 \omega \varphi)+c \cos (2 \omega \varphi)\right] \\
& D_{\varphi}^{C R}(\varphi)=\frac{\sigma^{2}}{2 \omega}\left[c^{2} \sin ^{2}(\omega \varphi)-c \sin (2 \omega \varphi)+\cos ^{2}(\omega \varphi)\right] .
\end{aligned}
$$

The corresponding quantities for the AR system are 


$$
\begin{aligned}
& I_{\varphi}^{A R}(\varphi)=1-\frac{\sigma^{2}}{2}\left[\frac{c}{2} \sin ^{2}(\omega \varphi)+\frac{1}{\omega} \sin (2 \omega \varphi)\right] \\
& V_{\varphi}^{A R}(\varphi)=1-\frac{\sigma^{2}}{4} c \sin ^{2}(\omega \varphi) \\
& D_{\varphi}^{A R}(\varphi)=\frac{\sigma^{2}}{2 \omega} \cos ^{2}(\omega \varphi) .
\end{aligned}
$$

Figure 4 plots the above quantities along with the stationary density of the phase reduced system (numerical solution of equation (3.7)) and the stationary density computed from Monte-Carlo simulations of the full system (3.4). Note the excellent agreement between the phase reduction and the full system (compare open circle to the solid lines in the lower panels). The CR (AR) system is shown in Fig. 4 (a) and (c) ((b) and (d)) with two different values for $c$ and all other parameters held constant. Recall from section 2 that increases in the parameter $c$ causes the oppositely-rotating region(s) of the vector field to move closer to the limit cycle (i.e., increasing $c$ brings $\rho_{*}$ closer to 1 ). It can clearly be seen that, in both systems, increasing the value of $c$ also causes the Fickian drift to become negative which signals the onset of the metastable switching behavior. Furthermore, the two points where $V_{\varphi}$ changes sign from positive to negative (see upper panels of Fig. 4 (c) and (d)) as $\varphi$ is increased are local maxima of the stationary density, where the oscillator spends most of its time, struggling to move past a dynamical barrier imposed by the noise. Thus, the zeros of the Fickian drift that have negative slope are metastable points that the system switches between. Note that the precise emergence of the metastable points would be difficult to discern from the stationary density alone as the two clear peaks in Figure 4 (c) and (d) are already somewhat apparent in Figure 4 (a) and (b). This is somewhat analagous to the dynamical behavior observed near the "ghost" of a saddle-node bifurcation, where deterministic trajectories slow down. Thus, the phase-reduced Fickian drift allows us to determine precisely when these metastable points emerge in the full system. It is important to note that the noise-induced contributions to the Fickian drift (the $\sigma^{2}$ terms) are what causes it to become negative. Indeed, if $\sigma$ is close to zero, then $V_{\varphi} \approx 1$ for both systems.

We can already see interesting differences between the two systems emerge in their respective phase reductions. In the AR system, the diffusivity is independent of $c$ which is not the case for the CR system. Thus, in the CR system, changing the dynamics of the vector field around the limit cycle (by varying c) affects both the drift and diffusivity of the phase reduced system, whereas in the AR system, the fluctuations of the phase variable are unaffected by the dynamics off the limit cycle (compare the dashed traces). It can also be seen that in the AR system, the diffusivity is small compared to the magnitude of the drift (compare the dashed lines to the solid lines in the upper panels of Fig. 4 (b) and (d)). Lastly, in the AR system, the the Ito and Fickian drifts are approximately the same whereas in the SL system, they are quite different (compare the dash dotted lines to the solid lines in the upper panels of Fig. 4).

Thus, the noise interacts with the nonlinear dynamics of the CR and AR systems in different ways to produce stochastic phase variables with qualitatively similar stationary behavior. However, recalling the trajectories shown in Figure 3, and noting the differences in the shapes of the stationary densities in the lower panels of Figure 4 (c) and (d), we can begin to see that there may be stronger differences in the transient behavior of the $\mathrm{CR}$ and $\mathrm{AR}$ systems. Indeed, if we plot stochastic phase trajectories from simulations of the full system (3.4) as in Figure 5, we can see strong differences emerging between the two systems. The first thing to note is that the switching behavior observed in Cartesian coordinates translates to sudden jumps in the trajectory of $\varphi$ that we will refer to as "phase jumps". The CR system displays fairly frequent phase jumps whereas in the AR system, these transitions are rare (compare the $\mathrm{x}$-axis scale in Fig. 5 (a) and (b)). This is similar to what we observed in simulations of the Cartesian system in Figure 3. Secondly, in the CR system, the phase jumps are in the positive phase direction (the same direction as the deterministic limit cycle) but in the AR system the jumps are in the opposite direction of the deterministic limit cycle.

We now seek to understand why the metastable behavior differs between the two systems. We will first concentrate on the different switching directions. In particular, we will show that the stationary 
probabilty flux is related to the directionality of the rotation of the oscillators, and we will derive analytical approximations to the flux in order to gain insight into the mechanism underlying the different switching directions.

\section{Stationary Probability Flux Determines Switching Direction}

Here, we derive analytical approximations to the flux for the AR and CR systems. For both systems, the metastable behavior increases as $c$ and $\sigma$ are varied such that the Fickian drifts becomes more negative. Because of this ambiguity, we introduce different scalings for the two systems in order to arrive at a single parameter which, when increased, causes metastable switching to occur. With this scaling, we are also able to take a distiguished limit $(c \rightarrow \infty$ and $\sigma \rightarrow 0)$ of the phase reduced systems in order to obtain analytically tractable approximations. We note that if $c$ becomes too large, then the phase reduction itself may cease being an accurate description of the full system. However, as long as we assume $\gamma>c \gg 1$, then the results in the next section hold, and we verify this fact with the use of numerical simulations.

\subsection{CR System}

We first introduce the parameters $\varepsilon=\frac{1}{c}$ and $K_{C R}=\frac{\sigma}{\varepsilon}$. Keeping the parameter $K_{C R}$ constant as we simultaneously let $\sigma \rightarrow 0$ and $\varepsilon \rightarrow 0$ allows us to expand the system in an asymptotic series in $\varepsilon$. To see this, substitute $c=\frac{1}{\varepsilon}$ and $\sigma=\varepsilon K_{C R}$ into equation (3.9) to obtain

$$
\begin{aligned}
I_{\varphi}^{C R}(\varphi) & =1-\varepsilon \frac{K_{C R}^{2}}{2 \omega} \cos (2 \omega \varphi)-\varepsilon^{2} \frac{K_{C R}^{2}}{2 \omega} \sin (2 \omega \varphi) \\
& \equiv I_{\varphi}^{C R, 0}(\varphi)+\varepsilon I_{\varphi}^{C R, 1}(\varphi)+\mathcal{O}\left(\varepsilon^{2}\right) \\
V_{\varphi}^{C R}(\varphi) & =1-\frac{K_{C R}^{2}}{2 \omega} \sin (2 \omega \varphi)+\varepsilon \frac{K_{C R}^{2}}{2 \omega} \cos (2 \omega \varphi) \\
& \equiv V_{\varphi}^{C R, 0}(\varphi)+\varepsilon V_{\varphi}^{C R, 1}(\varphi) \\
D_{\varphi}^{C R}(\varphi) & =\frac{K_{C R}^{2}}{2 \omega} \sin ^{2}(\omega \varphi)-\varepsilon \frac{K_{C R}^{2}}{2 \omega} \sin (2 \omega \varphi)+\varepsilon^{2} \frac{K_{C R}^{2}}{2 \omega} \cos ^{2}(\omega \varphi) \\
& \equiv D_{\varphi}^{C R, 0}(\varphi)+\varepsilon D_{\varphi}^{C R, 1}(\varphi)+\mathcal{O}\left(\varepsilon^{2}\right) .
\end{aligned}
$$

Next, we expand the stationary density $u_{s s}=u_{s s}^{0}+u_{s s}^{1}+\mathcal{O}\left(\varepsilon^{2}\right)$ to obtain an asymptotic approximation to the flux (3.8)

$$
J=\frac{1}{2 \pi / \omega}\left[1+\varepsilon \int_{0}^{2 \pi / \omega} I_{\varphi}^{C R, 1}(\varphi) u_{s s}^{0}(\varphi) d \varphi\right]+\mathcal{O}\left(\varepsilon^{2}\right),
$$

where we used the fact that $I_{\varphi}^{C R, 0}(\varphi)=1, \int_{0}^{2 \pi / \omega} u_{s s}^{0}(\varphi) d \varphi=1$, and $\int_{0}^{2 \pi / \omega} u_{s s}^{1}(\varphi) d \varphi=0$. Thus, in order to close our approximation, we must find the leading order approximation to the stationary density $u_{s s}^{0}$.

Starting from the Fickian form of the FP equation (3.7), we integrate once and expand terms in powers of $\varepsilon$ to obtain the leading order equation

$$
-J^{0}=D_{\varphi}^{C R, 0}(\varphi) \partial_{\varphi} u_{s s}^{0}-V_{\varphi}^{C R, 0}(\varphi) u_{s s}^{0}
$$

with $u_{s s}^{0}(0)=u_{s s}^{0}(2 \pi / \omega)$ and $J^{0}=(2 \pi / \omega)^{-1}$ is the leading order approximation to the flux. Owing to the simple forms of $V_{\varphi}^{C R, 0}$ and $D_{\varphi}^{C R, 0}$, we integrate the above equation and apply the boundary conditions to obtain

$$
u_{s s}^{0}=\frac{1}{2 \pi / \omega} \frac{i \omega}{K_{C R}^{2}} e^{-\Phi(\varphi)}\left[e^{A i} \operatorname{Ei}(-A \cot (\omega \varphi)+A i)-e^{-A i} \operatorname{Ei}(-A \cot (\omega \varphi)-A i)\right],
$$


where $A=2 \omega / K_{C R}^{2}$,

$$
\Phi(\varphi) \equiv \int \frac{-V_{\varphi}^{C R, 0}(\tilde{\varphi})}{D_{\varphi}^{C R, 0}(\tilde{\varphi})} d \tilde{\varphi}=\ln \left(\sin ^{2}(\omega \varphi)\right)+A \cot (\omega \varphi),
$$

and $\operatorname{Ei}(x)$ is the exponential integral. Figure 6 plots approximation $u_{s s}^{0}(4.3)$ against Monte-Carlo simulations of the full model (3.4) for three different values of $K_{C R}$. Notice that bistability increases as $K_{C R}$ is increased and that our approximation is in excellent agreement with the simulations for all values of $K_{C R}$. Thus, bistability occurs when the product of $c \sigma$ is large enough, illustrating that the noise interacts with the vector field in a nonlinear way in order to produce the metastable switching behavior.

Now that we have an approximation to the stationary density, we can compute our approximation to the stationary flux (4.2). Unfortunately, the integral in equation (4.2) can not be computed analytically, and we therefore approximate it using quadrature. Figure 7 plots the approximation to the flux (solid line) along with the results from Monte-Carlo simulations of the full system (3.4). The dashed line is $(2 \pi / \omega)^{-1}$ which is the leading order approximation to the flux. The lower panels plot example phase trajectories for three different values of $K_{C R}$. To compute the flux numerically, we took advantage of the fact that the system is ergodic and simulated (3.4) for a long time $T$. Using the time series $\varphi(t)$ obtained from the simulation, we then computed the time average of the Ito drift

$$
J=\frac{1}{2 \pi / \omega} \frac{1}{T} \int_{0}^{T} I_{\varphi}^{C R}(\varphi(t)) d t .
$$

It can clearly be seen that the simulations are in excellent agreement with our approximation. As $K_{C R}$ is increased, the flux decreases and eventually becomes negative. The value of $K_{C R}$ where the flux becomes negative is also the value of $K_{C R}$ where the Ito drift starts to become negative. Ignoring the $\varepsilon^{2}$ terms in $I_{\varphi}^{C R}(4.1)$, we find that the value of $K_{C R}$ where the Ito drift first becomes negative is given by $^{1} K_{C R}=\sqrt{2 \omega / \varepsilon}$ which is approximately 6.32 for the parameters in Figure 7 . Thus, we can see that around this point, the flux is close to zero and eventually becomes negative as $K_{C R}$ is increased further.

When the flux is positive, the phase jumps in the direction of the deterministic limit cycle (see bottom left panel in Figure 7). This explains the behavior we saw in Figure 5 (a) as $K_{C R}=2$ for the parameters used. However, when the flux is close to zero, the phase still jumps fairly regularly, however the jumps appear to be equally likely in both the postive and negative directions (bottom middle panel in Figure 7). Lastly, when the flux becomes negative, the process jumps in the direction opposite of the motion of the deterministic limit cycle (bottom right panel in Figure 7). Thus, it is clear that for the CR system, the flux determines the average direction of the phase jumps, but does not influence the rate at which the process jumps.

\subsection{AR System}

As in the previous section, we introduce the parameters $\varepsilon=\frac{1}{c}$ and $K_{A R}=\frac{\sigma^{2}}{\varepsilon}$. Note the difference between $K_{C R}$ and $K_{A R}$. Substituting $c=\frac{1}{\varepsilon}$ and $\sigma^{2}=\varepsilon K_{A R}$ into equation (3.10), we obtain

$$
\begin{aligned}
I_{\varphi}^{A R}(\varphi) & =1-\frac{K_{A R}}{4} \sin ^{2}(\omega \varphi)-\varepsilon \frac{K_{A R}}{2 \omega} \sin (2 \omega \varphi) \\
V_{\varphi}^{A R}(\varphi) & =1-\frac{K_{A R}}{4} \sin ^{2}(\omega \varphi) \\
D_{\varphi}^{A R}(\varphi) & =\varepsilon \frac{K_{A R}}{2 \omega} \cos ^{2}(\omega \varphi) .
\end{aligned}
$$

With this scaling, it is very easy to see that $I_{\varphi}^{A R} \approx V_{\varphi}^{A R}$ and that $D_{\varphi}^{A R} \approx 0$. Using equation (3.7), and noting that the leading order diffusivity is 0 , we obtain a very simple equation for the leading order

${ }^{1} \operatorname{Set} \min _{\varphi} I_{\varphi}^{C R, 0}(\varphi)+I_{\varphi}^{C R, 1}(\varphi)=1-\varepsilon \frac{K_{C R}^{2}}{2 \omega}=0$ and solve for $K_{C R}$. 
stationary density $u_{s s}^{0}$

$$
u_{s s}^{0}(\varphi)=\frac{J_{0}}{V_{\varphi}^{A R}(\varphi)} .
$$

From the above equation, we can see that the leading order flux is the same as the normalization constant for the density. Enforcing that $\int_{0}^{2 \pi / \omega} u_{s s}^{0}(\varphi) d \varphi=1$ we find

$$
J_{0}=\frac{\sqrt{4-K_{A R}}}{4 \pi / \omega} .
$$

However, notice that this approximation is only valid for $K_{A R}<4$. This is an interesting point that we will return to in the next section. Figure 8 plots our approximation $u_{s s}^{0}$ against Monte-Carlo simulations of the full model (3.4) for three different values of $K_{A R}$. Again, notice that bistability increases as $K_{A R}$ is increased. However, notice that our approximation begins to break down near $K_{A R}=4$ (Fig. 8 (c)), but this can be alleviated by taking $\gamma$ to be an order of magnitude larger (compare circles to crosses). This change in the behavior of the system can be seen even more clearly when we plot the flux as a function of $K_{A R}$ as in Figure 8. In contrast to the CR system, the flux in the AR system quickly goes to zero as $K_{A R}$ is increased. We can see that the analytical approximation works very well for values of $K_{A R}$ away from 4 (compare circles to solid line). Taken together with Figure 8 (c), this shows that, near $K_{A R}=4$, the $\mathcal{O}(1 / \gamma)$ terms that we are ignoring in the phase reduction (3.5) become significant. After $K_{A R}=4$, our approximation (4.6) is no longer valid. However, numerical simulations show that the flux is very close to zero.

As with the CR system, we can see that the flux is a good indicator of the average direction of the phase jumps (see the bottom panels in Fig. 9). However, it is difficult to interpret the results in the $K_{A R}>4$ regime as the flux is very close to zero. In contrast to the CR system, the rate of switching in the AR system also decreases with $K_{A R}$. Indeed, at $K_{A R}=3.5$, the system displays frequent phase jumps in the positive direction, but at $K_{A R}=6$ the system displays incredibly rare jumps in the negative direction (compare $\mathrm{x}$-axis scales in lower left and lower right panels) which is similar to the behavior we saw in Figure 5 (b) as $K_{A R}=6$ for the parameters used. However, this decrease in the jump rate cannot be due to the flux, as we saw that with near zero flux in the CR system, the rate of switching was still fairly frequent. In the next section, we seek to explain why there are differences in the switching frequency.

\section{Switching Frequency is Determined by Diffusivity}

We have shown that the onset of metastable switching in the CR and AR systems occurs when the Fickian drift becomes negative, and that the direction of the switching (either in the same or opposite direction of the deterministic limit cycle) is indicated by the sign of the stationary probability flux. Here we will show that the frequency of the switching events can be explained by the strength of the stochastic fluctuations in the phase variable (3.5).

\subsection{Rare Switching in AR System is Caused by Weak Diffusivity}

To understand both the decreasing rate of switching and the change in switching direction of the AR system, we examine the contributions of the Ito drift and diffusivity to the reduced phase model using the scalings introduced in the previous section. First, recall the diffusivity for the AR model given in (4.5). Notice two things about the AR diffusivity: (1) that it is order $\varepsilon$; and (2) that it has two zeros $\varphi^{*}=\frac{\pi}{2 \omega}, \frac{3 \pi}{2 \omega}$ regardless of the values of $K_{A R}$ and $\varepsilon$. We will refer to these zeros of the diffusivity as "ratchet points". The only way that the reduced phase system can cross these ratchet point is if the Ito drift is nonzero at these points. Figure 10 (a) plots the Ito drift (solid) and diffusivity (dashed) as a function of $\varphi$ and (b) plots a sample stochastic phase trajectory of the full system (3.4) when $K_{A R}=3.5$. In this case, the nonlinear dynamics of the full model filter the noise so that it is $\mathcal{O}(\varepsilon)$ in the phase reduced system. Thus, the Ito drift of the phase reduced system is the main determinant of the motion 
of the stochastic process. This can be seen in Fig. 10 (b) where the phase variable increases steadily over time. The effect of the changing Ito drift can be seen in the slope of the trajectory as the phase variable increases more slowly when it is near the two ratchet points. In this case, the Ito drift is positive at the ratchet points (as indicated by the horizontal arrows) which indicates that the stochastic trajectory will pass over the ratchet points in the direction of the deterministic limit cycle.

As $K_{A R}$ is increased to 3.961 as in Fig. 10 (c), the Ito drift near the ratchet points becomes the same order at the diffusivity (inset). Thus, the process begins to have a more difficult time passing over the ratchet points, and begins to display the phase jumps seen previously (Fig. 10 (d)). The jumps occur in the positive direction as the Ito drift at the ratchet point is still positive (horizontal arrows). When $K_{A R}=4$ (Fig. $10(\mathrm{e})$ ), an interesting phenomenon occurs: the Ito drift is exactly zero at the ratchet points (to see this, plug $\varphi^{*}=\frac{\pi}{2 \omega}, \frac{3 \pi}{2 \omega}$ into the equation for $I_{\varphi}^{A R}$ in (4.5)). Thus, the phase reduced system (3.5) predicts that once a trajectory arrives at a ratchet point, it will be stuck there indefinitely. However, it is clear from the $\gamma=50$ sample trajectory in Fig. 10 (f) that the $\mathcal{O}(1 / \gamma)$ terms in the phase reduction become significant as the trajectory of the full system (3.4) is able to pass over the ratchet points. This discrepency between the full system and the phase reduced system is caused by the fact that the phase reduction ignores amplitude fluctuations. That is, for these values of $K_{A R}$ and $\gamma$, fluctuations in the amplitude variable allow the full system (3.4) to move past the ratchet points. However, as $\gamma$ is increased, the trajectory has a more difficult time overcoming the ratchet points $(\gamma=500$ and $\gamma=5000$ traces). Thus, as $\gamma \rightarrow \infty$ the stationary density for $\varphi$ converges to a delta function at one of the two ratchet points depending on the initial conditions.

Lastly, when $K_{A R}>4$ (Fig. $10(\mathrm{~g})$ ), the Ito drift becomes negative at the ratchet points, and the phase reduction predicts that the process can only move past the ratchet points in the opposite direction of the deterministic limit cycle. However, the Ito drift is only negative in a neighborhood of the ratchet points, and is strongly positive everywhere else. Furthermore, the diffusivity is still much smaller than the Ito drift. Thus, away from the ratchet points, the Ito drift dictates that the process move in the same direction of the limit cycle. Therefore, the phase reduction predicts that the only way that the process can jump between ratchet points is via a rare event where the noise overcomes the positive Ito drift and the process jumps in the opposite direction of the deterministic limit cycle (Fig. 10 (h), $\gamma=50$ trace). Thus, the rare switching in the AR system is caused by the fact that the diffusivity is weak and that the Ito drift is negative near the ratchet points, but positive away from the ratchet points. In contrast to the phase reduced system, if $\gamma$ is made smaller, fluctuations in the amplitude variable allow the full system (3.4) to pass over the ratchet points in the same direction of the deterministic limit cycle (right panel, $\gamma=10$ trace). Note also that the small diffusivity is the reason why our asymptotic approximation (4.6) breaks down when $K_{A R}>4$. That is, our approximation assumes that the diffusivity is 0 for all $\varphi$. However, close to the points where the Ito drift first becomes negative, the diffusivity is non-negligible (and is in fact the reason why a switch can actually occur).

We can quantify the accuracy of the phase reduced system (3.5) by computing the $L^{2}$ norm of the difference between the stationary density obtained from solving the FP equation for the reduced system (3.6) $u_{s s}$ and the stationary density obtained from Monte-Carlo simulation $u_{s s}^{M C}$. More precisely, we take the error to be

$$
\text { Error }=\left(\int_{0}^{2 \pi / \omega}\left[u_{s s}(\varphi)-u_{s s}^{M C}(\varphi)\right]^{2} d \varphi\right)^{1 / 2} .
$$

Figure 11 plots this error as a function of $K_{A R}$ and two different values for $\gamma$. Note that to solve (3.6), we found it best to use a forward finite difference scheme when the flux was positive, and a backwards finite difference scheme when the flux was negative. It is clear that the phase reduced model is highly accurate for $K_{A R}<4$, but the error quickly increases as $K_{A R} \rightarrow 4_{+}$. Interestingly, the $\mathcal{O}(1 / \gamma)$ terms appear to be more important for accuracy when $K_{A R}>4$. Regardless, increasing $\gamma$ causes the error to decrease for all values $K_{A R} \neq 4$ (compare solid and dashed lines). 


\subsection{Faster Switching in the CR System is Caused by Strong Diffusivity}

For the CR system, the ratchet points (zeros of the diffusivity) occur at approximately $\varphi^{*}=0, \pi$ as $D_{\varphi}^{C R}\left(\varphi^{*}\right)=\mathcal{O}\left(\varepsilon^{2}\right)$ (see (4.1)) independent of $K_{C R}$. Figure 12 (a) plots the Ito drift and diffusivity for the CR system when $K_{C R}=4$. In this case, the diffusivity is much larger than the diffusivity in the AR system. Thus, switching between the metastable states (zeros of the Fickian drift with negative slope) will occur at a higher rate than in the AR system. At the ratchet points, the Ito drift is positive, which indicates that the reduced phase system (3.5) predicts that the process moves past the ratchet points in the direction of the deterministic limit cycle. A sample trajectory from the full system (3.4) in Fig. 12 (b) shows that this is indeed the case as $\varphi$ increases with time.

Figure 12 (c) shows the behavior of the CR system when $K_{C R}=\sqrt{2 \omega / \varepsilon} \approx 6.32$ where $I_{\varphi}^{C R}\left(\varphi^{*}\right)=$ $\mathcal{O}\left(\varepsilon^{2}\right)$ (see (4.1)). Thus, the Ito drift is approximately zero at the ratchet points. As in the AR system, the phase reduction predicts that once a trajectory arrives at a ratchet point, it will be stuck there indefinitely. However, the sample trajectories (Fig. 12 (d)) again show that accuracy of this prediction is highly dependent on the value of $\gamma$. Lastly, when $K_{C R}>\sqrt{2 \omega / \varepsilon}$ (Fig. 12 (e)), the Ito drift becomes negative at the ratchet points, and the phase reduction predicts that the process can only move past the ratchet points in the opposite direction of the deterministic limit cycle. In contrast to the AR system, the CR system has a large diffusivity (away from the ratchet points). Thus, the switching in the opposite direction on the limit cycle will occur at a higher rate than in the AR system (compare Fig. 12 (f) to Fig. $10(\mathrm{~h})$ ). Just as in the AR system, if $\gamma$ is made smaller, the full system can also overcome the ratchet points in the same direction of the deterministic limit cycle by moving off the limit cycle (right panel, $\gamma=10$ trace).

Just as we did with the AR system, we quantify the accuracy of the phase reduced system (3.5) by exploring the error (5.1) between the stationary density obtained from solving the FP equation for the reduced system (3.6) $u_{s s}$ and the stationary density obtained from Monte-Carlo simulation $u_{s s}^{M C}$. Figure 13 plots this error as a function of $K_{C R}$ and two different values for $\gamma$. Interestingly, the error overall appears to be larger than what we found in the AR system (compare y-axis scale in Figures 13 and 11). Nonetheless, the qualitative trend is the same in both systems: as $K_{C R}$ approaches the value where the Ito drift is zero at the ratchet points, the error in the phase reduction increases. Furthermore, increasing $\gamma$ causes the error to decrease for all values $K_{C R} \neq \sqrt{2 \omega / \varepsilon}$ (compare solid and dashed lines).

\section{Discussion}

We have shown that a limit cycle system with additive noise can display a wide range of metastable switching dynamics. Using stochastic phase reduction methods [28,31], we are able to relate different aspects of this metastable switching behavior to terms appearing in the phase reduced model. That is, the emergence of metastable switching in a limit cycle system occurs when the drift term of the conservation (Fickian) form of the Fokker-Planck equation for the phase reduced system becomes negative. The zeros of the Fickian drift with negative slope represent metastable points that the stochastic process switches between and correspond to peaks in the stationary density for the phase. The rate and directionality of the switching are controlled by the drift term in the stochastic differential equation for the phase reduced system, i.e., the Ito drift. We have shown that the Ito drift determines the stationary probability flux, and thereby the directionality of the switching. That is, if the flux is positive (negative), the system will (on average) switch between the metastable points in the same (opposite) direction of the deterministic limit cycle. Lastly, as with the Kramer's problem, the magnitude of the diffusivity determines the rate at which the process switches between the metastable points.

We illustrate these findings with the use of simple planar limit cycle systems that have areas in their vector fields where rotation occurs in the opposite direction of the limit cycle. In the CR system, this counterrotation occurs on only one side of the limit cycle. The effect of this counterrotation is to amplify the noise in the phase variable. This leads to a large diffusivity in the phase reduced system. Thus, the CR system displays fairly frequent switching between metastable points. In contrast, the counterrotation in the AR system occurs both inside and outside of the limit cycle. The phase reduction for the AR system has a diffusivity that is very small $(\mathcal{O}(\varepsilon)$ ), and thus, switching between metastable points can 
be extremely rare. Both systems can display switching in either the same or opposite direction of the deterministic limit cycle. Thus, it is the differences in the dynamics off the limit cycle between the two systems that is responsible for the different rates of switching. This highlights the fact that in stochastic limit cycle systems, if the interactions of the noise with the vector field off the limit cycle are ignored in the phase reduction (i.e., the $\mathcal{O}\left(\sigma^{2}\right)$ terms in (3.5)), then the phase model will fail to capture many interesting phenomena that are present in the full system (2.1).

We have also identified an interesting singularity where the leading order phase reduced system fails to capture the dynamics of the full system regardless of the rate of attraction back to the limit cycle. This occurs when the Ito drift of the phase reduced system has a zero at exactly the same point as the diffusivity. In this case, the phase reduction predicts that the system should remain stuck at this point for all time. However, simulations of the full system reveal that this is not the case. Thus, $\mathcal{O}(1 / \gamma)$ terms that are ignored in the leading order reduction become necessary to capture the behavior of the full system.

Multiple peaks in the stationary densities for the phase differences of oscillators coupled through shared noise has been previously observed [19]. However, in contrast to our work, the authors of this study focused on the dynamics of multiple oscillators recieving both shared and independent noise. Depending on how the shared noise is input into the system (e.g., additive or multiplicative) they see different types of clustered stationary states, corresponding to different peaks in the stationary densities of the phase difference. An interesting avenue for future work is to explore how shared noise affects the clustering of oscillators that display the metastable switching we have examined here. However, one must be cautious when interpreting the stationary densities for the phase differences in the case of metastable switching. More precisely, if one examined the stationary density of the phase difference between two uncoupled oscillators with asymmetric noise that individually display metastable switching, the distribution would appear bimodal, with peaks around $0,2 \pi$ and $\pi$. This is due to the fact that both oscillators spend most of their time near the same two metastable points which are on opposite sides of the limit cycle. Thus, it would appear that individual noise causes the two oscillators to jump between synchrony and anti-phase.

This work identifies an alternative mechanism for the emergence of metastable switching dynamics observed in a number of physical systems ranging from the dynamics of genetic swiches in bacteria [10, $15,29]$ to up and down states in the cortex thought to be involved in working memory [4,8]. Interestingly, the dynamics of the phase variable in the CR system (Figure 5 (a)) is reminiscent of the behavior of a brownian ratchet, which is a standard model for molecular motors $[1,13,27]$. Furthermore, as this metastable switching behavior has also been shown to occur in more complex limit cycle systems [20], this could lead to interesting consequences for coupled oscillator systems. Indeed, an interesting type of mixed synchronization has already been observed when oppositely rotating oscillators are coupled together (see, for example, $[3,23]$ ). Exploring the behavior of coupled metastable oscillators could lead to new synchronization or phase-locking phenomena.

\section{Acknowledgements}

The authors are supported in part by the Mathematical Biosciences Institute and the National Science Foundation under Grant No. DMS-0931642.

\section{References}

[1] R.D. Astumian and M. Bier. Fluctuation driven ratchets: molecular motors. Phys. Rev. Lett., 72(11):1766, 1994. 
[2] T. Betz, D. Lim, and J.A. Käs. Neuronal growth: a bistable stochastic process. Phys. Rev. Lett., 96(9):098103, 2006.

[3] S.K. Bhowmick, D. Ghosh, and S.K. Dana. Synchronizaion in counter-rotating oscillators. Chaos, 21(3):033118, 2011.

[4] R. Cossart, D. Aronov, and R. Yuste. Attractor dynamics of network up states in the neocortex. Nature, 423(6937):283-288, 2003.

[5] REL DeVille, E Vanden-Eijnden, and CB Muratov. Two distinct mechanisms of coherence in randomly perturbed dynamical systems. Physical Review E, 72(3):031105, 2005.

[6] G.B. Ermentrout and D.H. Terman. Mathematical Foundations of Neuroscience. Springer-Verlag, Berlin, 2010.

[7] R. FitzHugh. Impulses and physiological states in theoretical models of nerve membrane. Biophys. J., 1:445-466, 1961.

[8] J.M. Fuster. Memory in the cerebral cortex. MIT Press, Cambridge, MA, 1996.

[9] C.W. Gardiner. Handbook of Stochastic Methods. Springer, NY, 1997.

[10] T.S. Gardner, C.R. Cantor, and J.J. Collins. Construction of a genetic toggle switch in escherichia coli. Nature, 403(6767):339-342, 2000.

[11] D. Gonze, J. Halloy, and P. Gaspard. Biochemical clocks and molecular noise: theoretical study of robustness factors. J. Chem. Phys., 116:10997-11010, 2002.

[12] H. Koeppl, M. Hafner, A. Ganguly, and A. Mehrotra. Deterministic characterization of phase noise in biomolecular oscillators. Phys. Biol., 8:055008, 2011.

[13] A.B. Kolomeisky and M.E. Fisher. Molecular motors: a theorist's perspective. Annu. Rev. Phys. Chem., 58:675-695, 2007.

[14] Y. Kuramoto. Chemical Oscillations, Waves, and Turbulence. Springer-Verlag, Berlin, 1984.

[15] K. Lai, M.J. Robertson, and D.V. Schaffer. The sonic hedgehog signaling system as a bistable genetic switch. Biophys. J., 86(5):2748-2757, 2004.

[16] P. Le Gal, A. Nadim, and M. Thompson. Hysteresis in the forced stuart-landau equation: application to vortex shedding from an oscillating cylinder. J. Fluid. Struct., 15:445-457, 2001. 
[17] B Lindner and L Schimansky-Geier. Analytical approach to the stochastic fitzhugh-nagumo system and coherence resonance. Phys Rev E, 60(6):7270, 1999.

[18] J. Nagumo, S. Arimoto, and S. Yoshizawa. An active pulse transmission line simulating nerve axon. Proc IRE., 50:2061-2070, 1962.

[19] H. Nakao, K. Arai, and Y. Kawamura. Noise-induced synchronization and clustering in ensembles of uncoupled limit-cycle oscillators. Phys. Rev. Lett., 98(18):184101, 2007.

[20] J.M. Newby and M.A. Schwemmer. Effects of moderate noise on a limit cycle oscillator: Counterrotation and bistability. Phys. Rev. Lett., 112(11):114101, 2014.

[21] A. Pikovsky, M. Rosenblum, and J. Kurths. Synchronization: A Universal Concept in Nonlinear Sciences. Cambridge University Press, Cambridge, 2001.

[22] AS Pikovsky and J Kurths. Coherence resonance in a noise-driven excitable system. Phys Rev Lett, $78(5): 775,1997$.

[23] A. Prasad. Universal occurrence of mixed synchronization in counter-rotating nonlinear coupled oscillators. Chaos Soliton Fract., 43(1):42-46, 2010.

[24] M. Provansal, C. Mathis, and L. Boyer. Bénard-von kármán instability: transient and forced regimes. J. Fluid Mech., 182:1-22, 1987.

[25] M.A. Schwemmer and T.J. Lewis. The Theory of Weakly Coupled Oscillators. In N.W. Schultheiss, A. Prinz, and R.J. Butera, editors, Phase Response Curves in Neuroscience: Theory, Experiment, and Analysis, pages 3-32. New York: Springer, 2012.

[26] J.T. Stuart. On the non-linear mechanics of wave disturbances in stable and unstable parallel flows. J. Fluid Mech., 9:353-370, 1960.

[27] K. Svoboda, C.F. Schmidt, B.J. Schnapp, and S.M. Block. Direct observation of kinesin stepping by optical trapping interferometry. Nature, 365(6448):721-727, 1993.

[28] J. Teramae, H. Nakao, and G.B. Ermentrout. Stochastic phase reduction for a general class of noisy limit cycle oscillators. Phys. Rev. Lett., 102:194102, 2009.

[29] J.-W. Veening, W.K. Smits, and O.P. Kuipers. Bistability, epigenetics, and bet-hedging in bacteria. Annu. Rev. Microbiol., 62:193-210, 2008.

[30] A.T. Winfree. The Geometry of Biological Time. Springer, New York, 2001. 
[31] K. Yoshimura and K. Arai. Phase reduction of stochastic limit cycle oscillators. Phys. Rev. Lett., 101:154101, 2008.

\section{Figures}

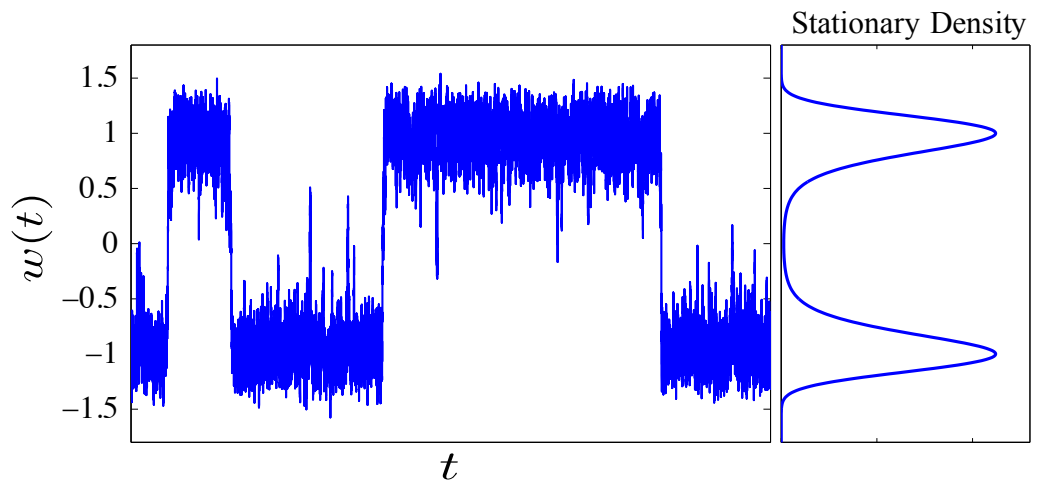

Figure 1: Metastable switching. The left panel shows a stochastic process $w(t)$ that spends most of its time near the deterministic stable fixed points \pm 1 . The right panel shows the stationary density for this process which has two distinct peaks.

\section{CR System}

(a)

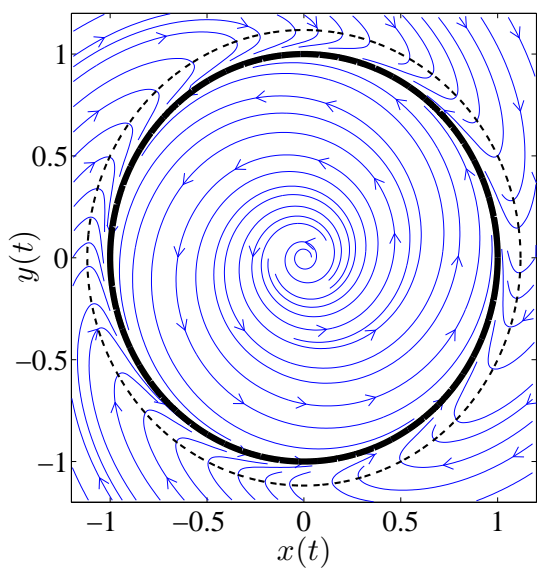

AR System

(b)

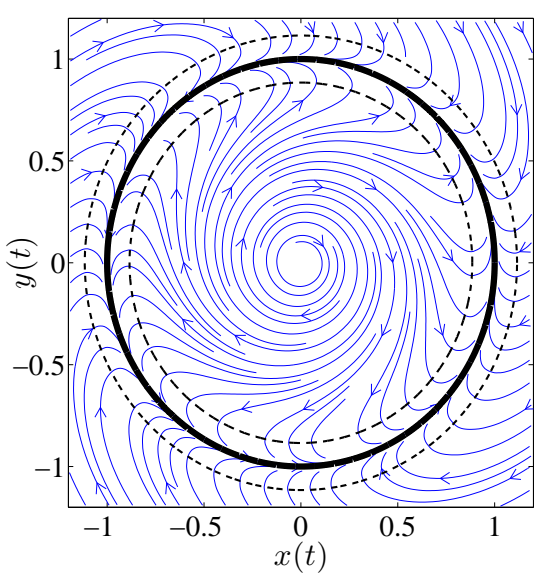

Figure 2: Vector fields of the two planar systems. Thin lines represent streamlines of the deterministic vector fields. Thick line is the limit cycle. (a) The counterrotating (CR) system with $c=4$ and $\gamma=1$. The vector field changes rotation direction at one value of $\rho$ outside of the limit cycle (dashed line). (b) The antirotating (AR) system with $c=15$ and $\gamma=5$. The vector field changes rotation direction at two values of $\rho$ one outside and one inside of the limit cycle (dashed lines). 
CR System

(a)
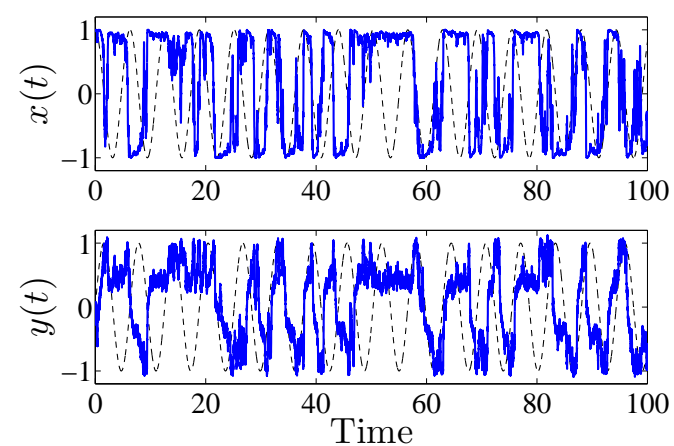

AR System

(b)
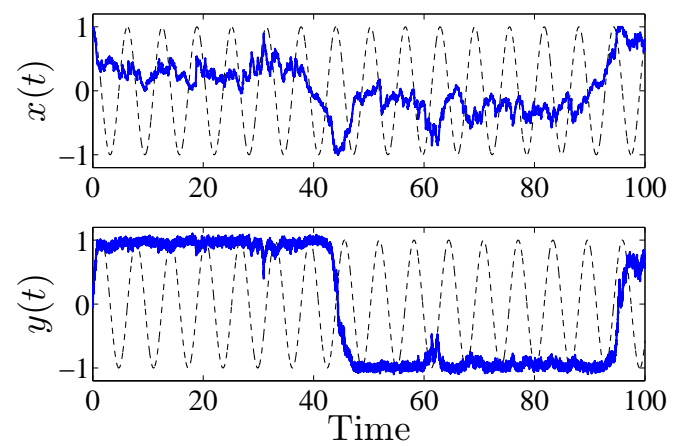

Figure 3: Metastable switching in the CR and AR systems. Dashed lines show the deterministic limit cycle $\sigma=0$ (which is the same in both cases). For each system $\gamma=50$ and $\omega=1$ (a) CR system with $c=4$ and $\sigma=0.5$. Metastable switching is most prominent in the x-component. (b) AR system with $c=20$ and $\sigma=0.45$. Metastable switching is most prominent in the y-component. 
CR System

(a)
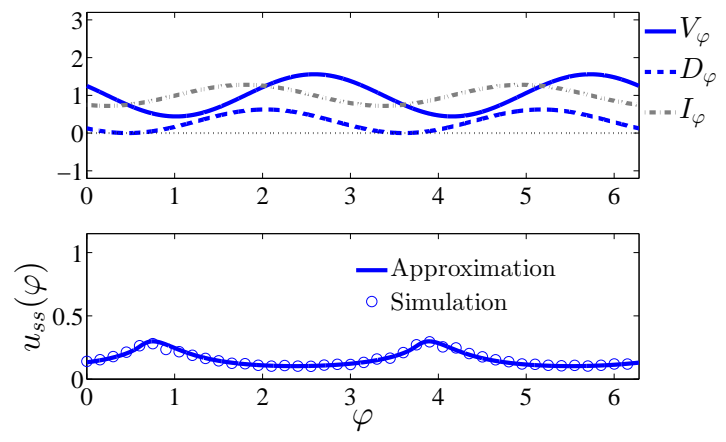

(c)
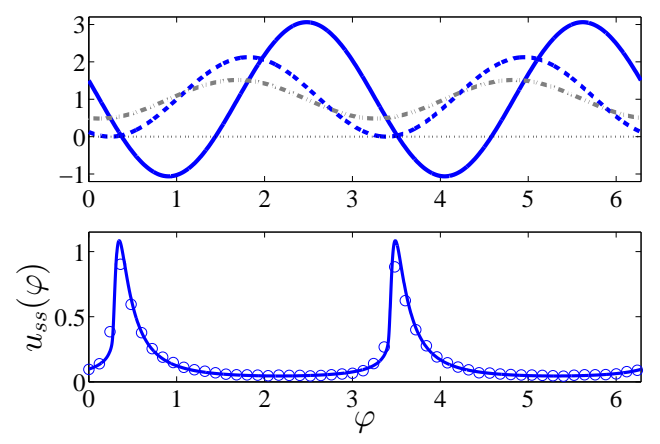

AR System

(b)
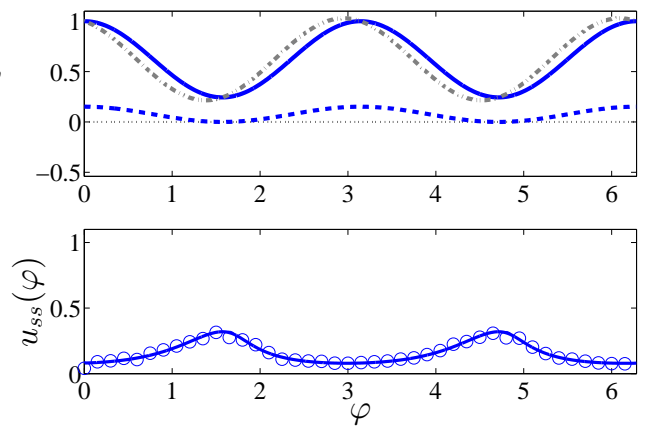

(d)
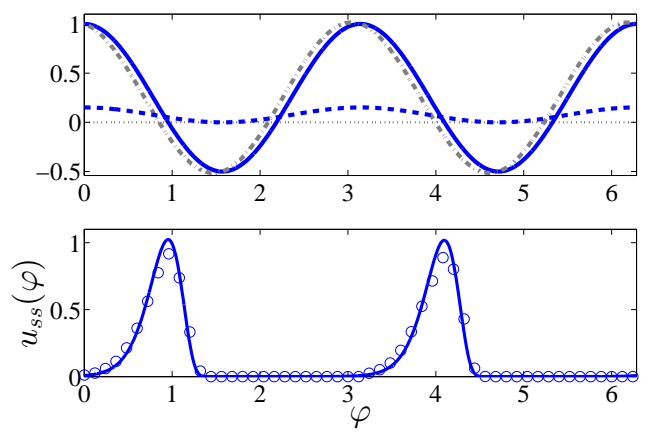

Figure 4: Bistability occurs when the Fickian drift becomes negative. Upper panels plot the Fickian drift $\left(V_{\varphi}\right.$, solid lines), Ito drift $\left(I_{\varphi}\right.$, grey dash-dotted lines), and diffusivity $\left(D_{\varphi}\right.$, solid lines) for the phase reduced system (3.5). Lower panels plot the stationary density of the phase reduced system (solid lines) obtained from solving (3.6) along with Monte-Carlo simulations of the full system (3.4) (open circles) with $\gamma=50$. In all cases, $\omega=1$. The (a) CR system with $c=2$ and $\sigma=0.5$ and the (b) AR system with $c=10$ and $\sigma=0.55$ both have relatively flat stationary densities. Strong peaks in the stationary densities occur when the Fickian drift crosses zero with a negative slope in (c) CR system with $c=4$ and $\sigma=0.5$ and (d) CR system with $c=20$ and $\sigma=0.5$. To generate the simulated histograms, we numerically solved (3.4) using the Euler-Mayurama method with $d t=0.001$ and simulation time $T=1,000$. 
(a)

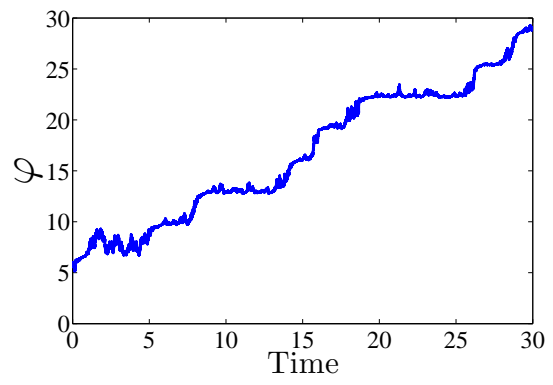

(b)

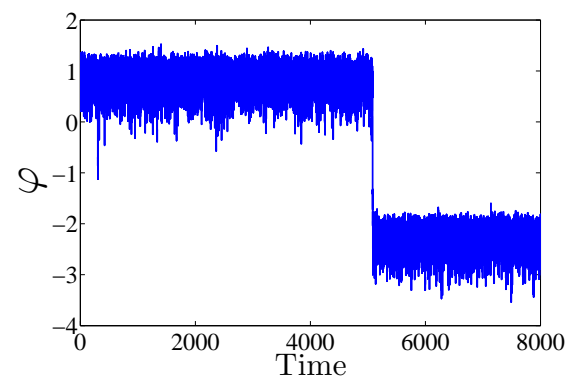

Figure 5: CR and AR systems display different switching dynamics. Sample phase trajectories from simulations of the full system (3.4) with $\gamma=50$ and $\omega=1$. (a) CR system with $c=4$ and $\sigma=0.5$ displays frequent switching while (b) AR system with $c=20$ and $\sigma=0.55$ displays very rare switiching (note the difference in $\mathrm{x}$-axis scale). Note that we are considering phase here without the modulo $2 \pi / \omega$ in order to better illustrate when phase is increasing/decreasing.

(a)

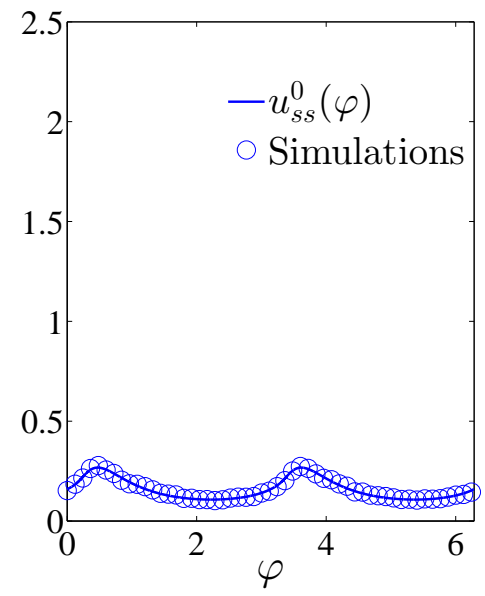

(b)

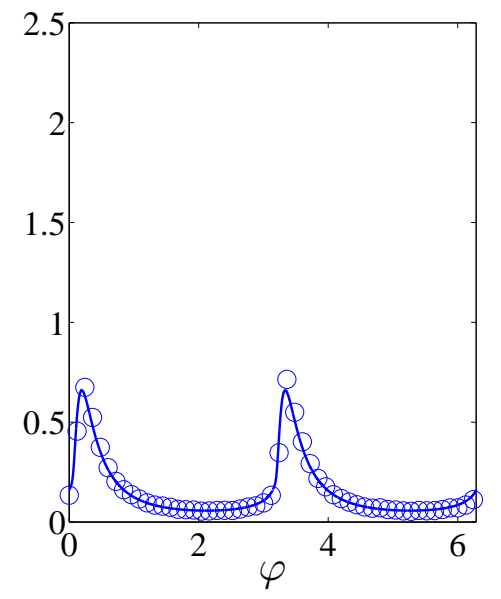

(c)



Figure 6: Approximations of the CR stationary density. The asymptotic approximation (4.3) (solid line) is plotted against Monte-Carlo simulations of the full system (3.4) (open circles) for (a) $K_{C R}=1$, (b) $K_{C R}=2$, and (c) $K_{C R}=4$. In all figures, $\gamma=50, c=20$, and $\omega=1$. To generate the simulated histograms, we numerically solved (3.4) using the Euler-Mayurama method with $d t=0.001$ and simulation time $T=1,000$. 


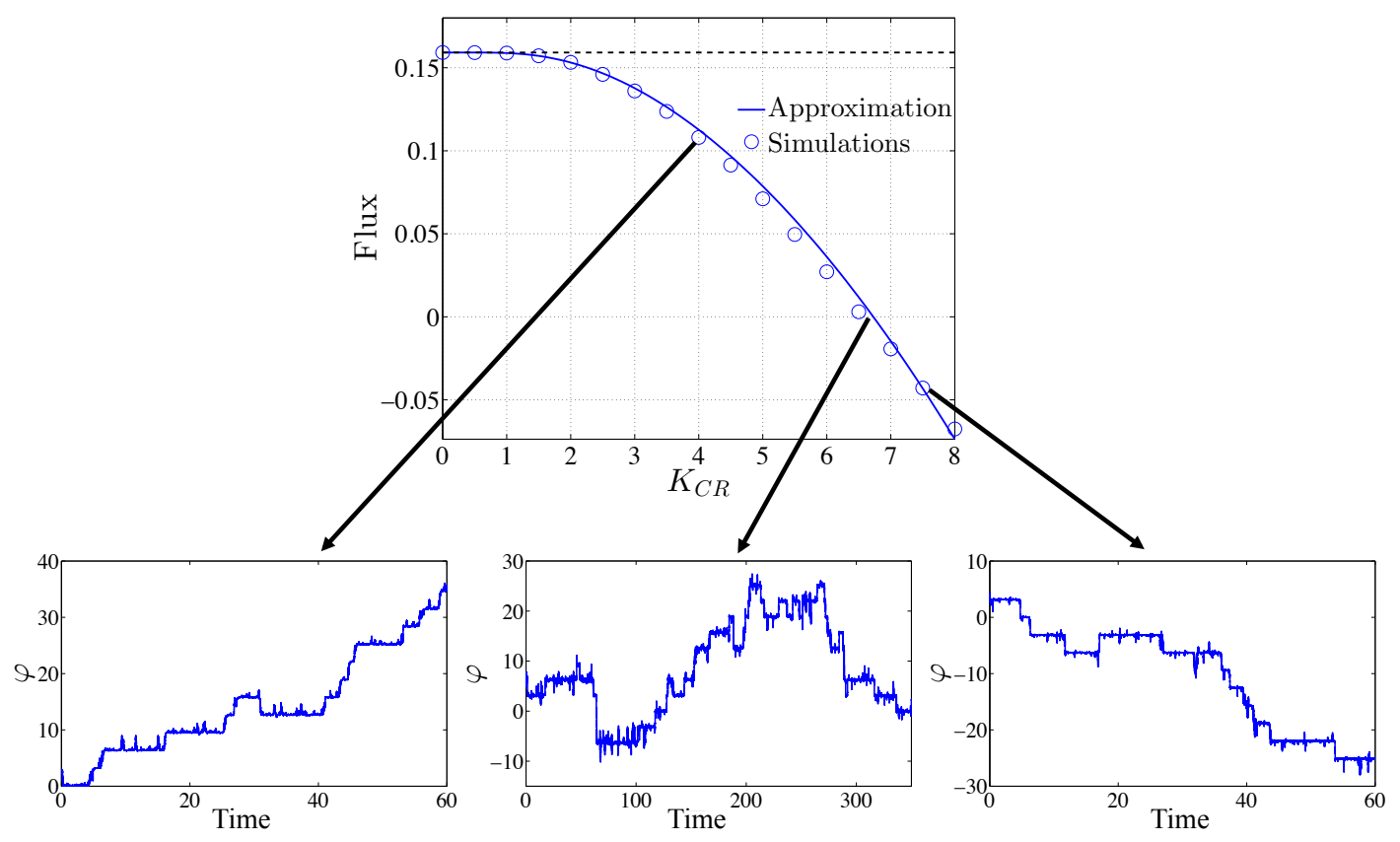

Figure 7: Approximation of the CR flux. The asymptotic approximation (4.2) (solid line) is plotted as a function of $K_{C R}$ along with simulations of the full system (3.4) open circles. The dashed line is $(2 \pi / \omega)^{-1}$ which is the frequency of the deterministic limit cycle and the zeroeth order approximation to the flux. Lower figures are sample phase trajectories of the full system (3.4). From left to right $K_{C R}=4, K_{C R}=6.6$, and $K_{C R}=7.5$. In all figures, $\gamma=50, c=20$, and $\omega=1$. To generate the flux, we numerically solved (3.4) using the Euler-Mayurama method with $d t=0.001$ and simulation time $T=1,000$ and plugged the phase trajectory into (4.4). Note that in the lower figures we are considering phase without the modulo $2 \pi / \omega$ in order to better illustrate when phase is increasing/decreasing. 
(a)

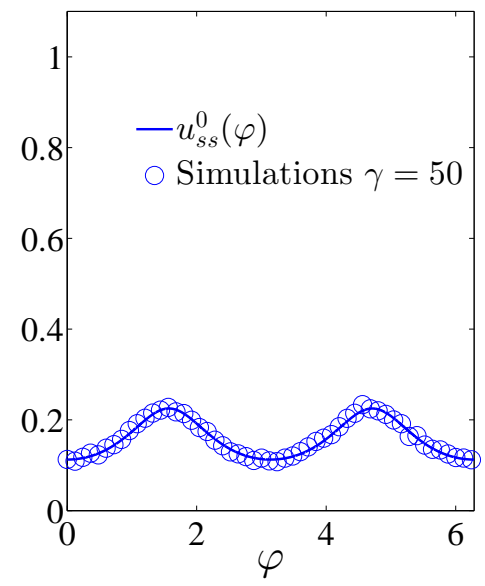

(b)

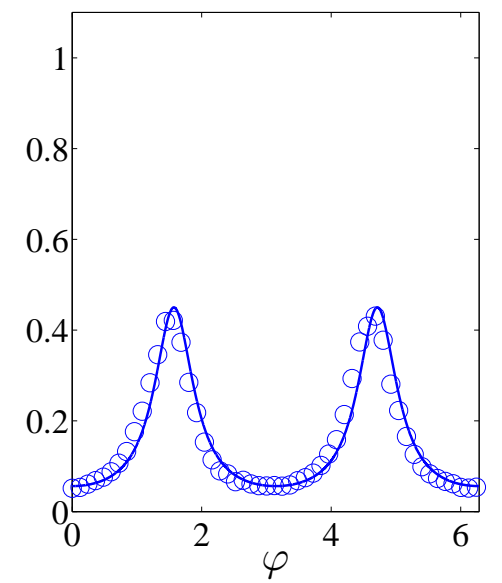

(c)

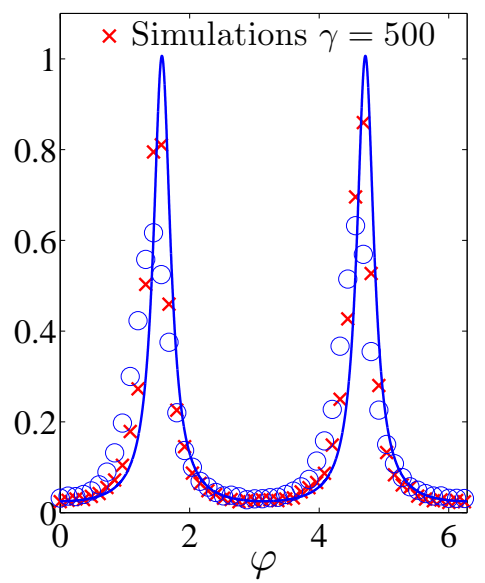

Figure 8: Approximations of the AR stationary density. The asymptotic approximation (4.6) (solid line) is plotted against Monte-Carlo simulations of the full system (3.4) (open circles) for (a) $K_{C R}=2$, (b) $K_{C R}=3.5$, and (c) $K_{C R}=3.9$. In all figures, $\gamma=50, c=20$, and $\omega=1$. The crosses in (c) are simulations of the full system with $\gamma=50$. To generate the simulated histograms, we numerically solved (3.4) using the Euler-Mayurama method with $d t=0.001$ and simulation time $T=3,000$. 


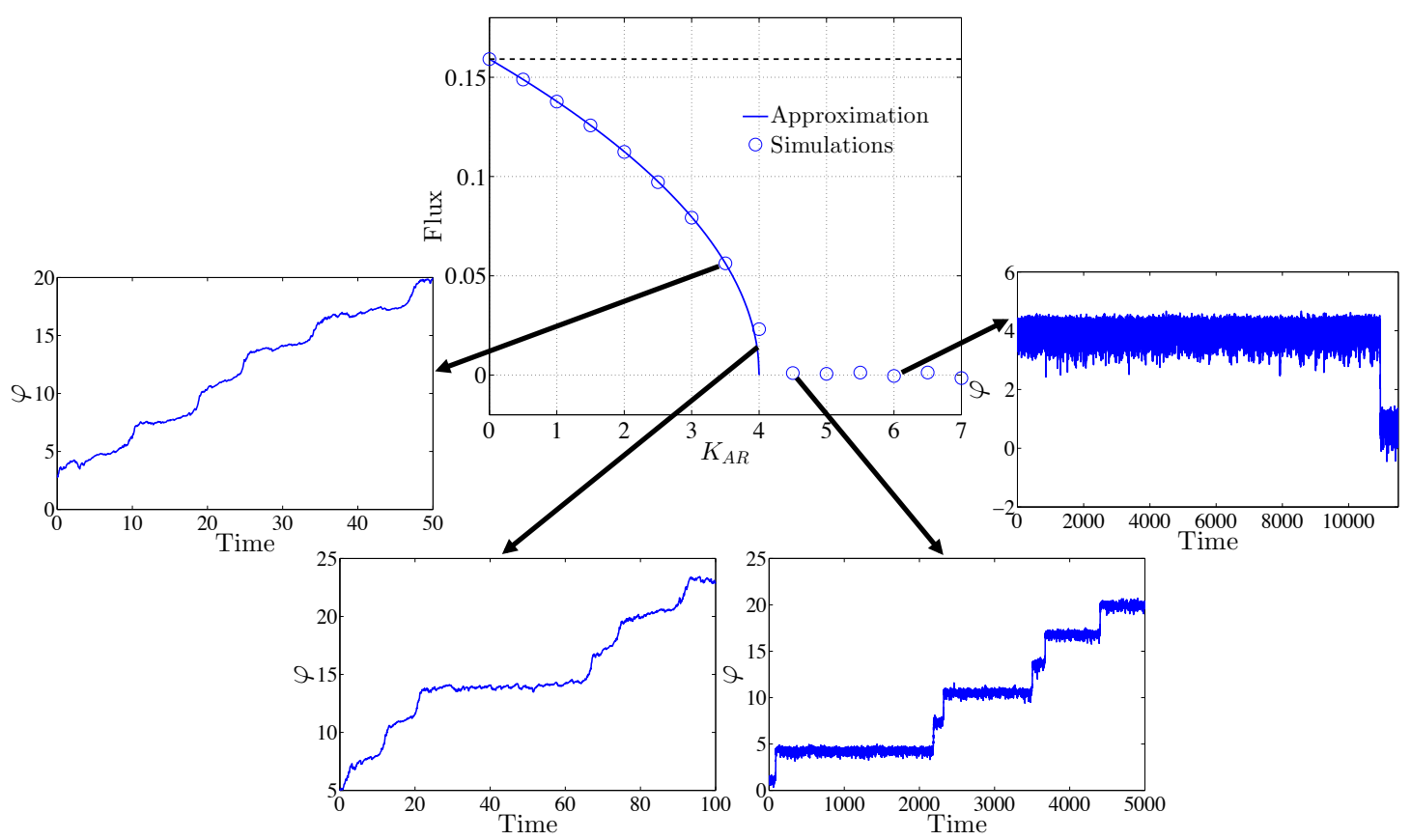

Figure 9: Approximation of the AR flux. The asymptotic approximation (4.7) (solid line) is plotted as a function of $K_{A R}$ along with simulations of the full system (3.4) open circles. The dashed line is $(2 \pi / \omega)^{-1}$ which is the frequency of the deterministic limit cycle. Lower figures are sample phase trajectories of the full system (3.4). From left to right $K_{A R}=3.5, K_{A R}=3.9, K_{A R}=4.5$, and $K_{A R}=6$. In all figures, $\gamma=50, c=20$, and $\omega=1$. To generate the flux, we numerically solved (3.4) using the Euler-Mayurama method with $d t=0.001$ and simulation time $T=1,000$ and plugged the phase trajectory into (4.4). Note that in the lower figures we are considering phase without the modulo $2 \pi / \omega$ in order to better illustrate when phase is increasing/decreasing. 
(a)

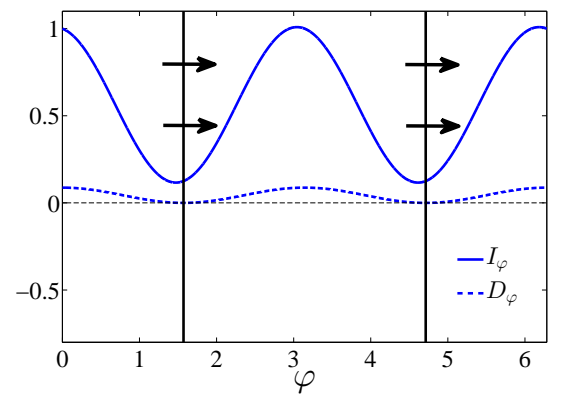

(c)

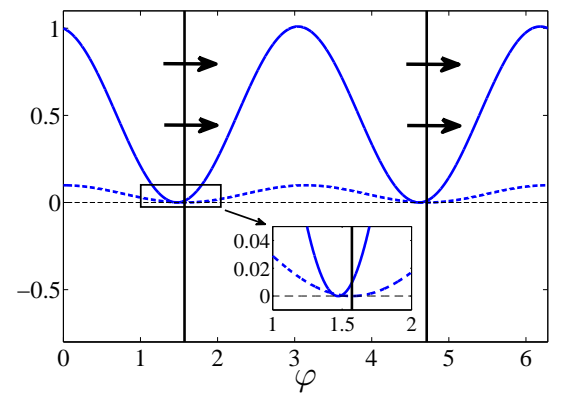

(e)

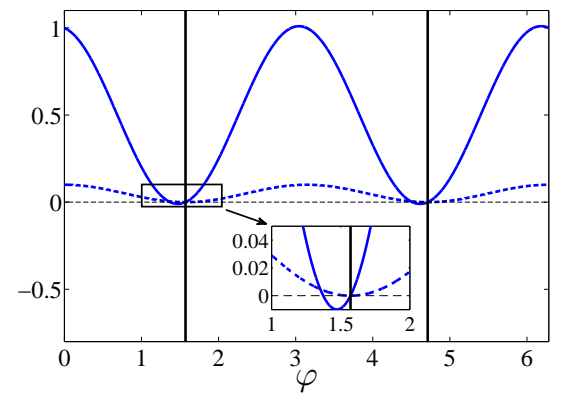

(g)

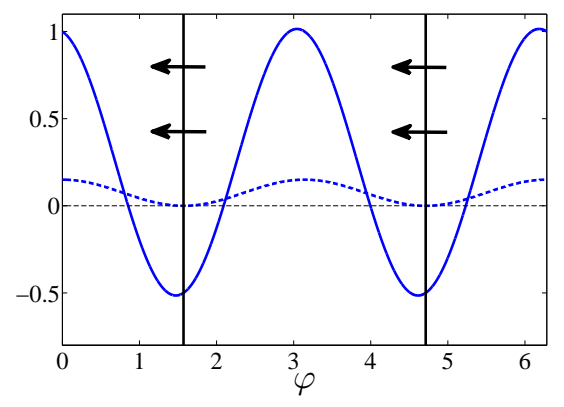

(b)

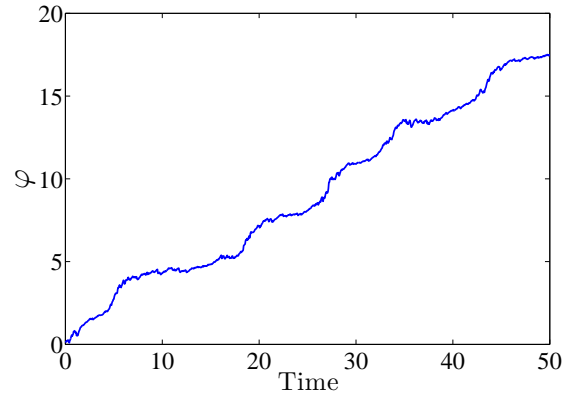

(d)

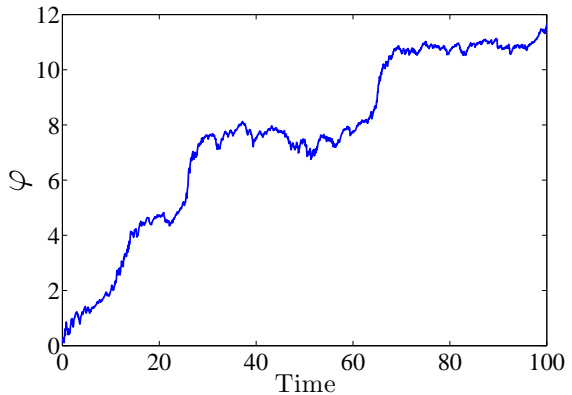

(f)

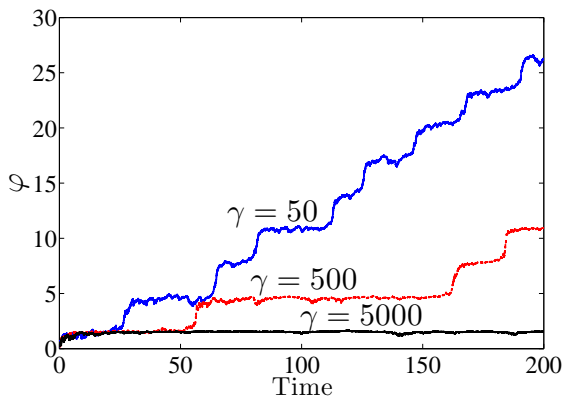

(h)

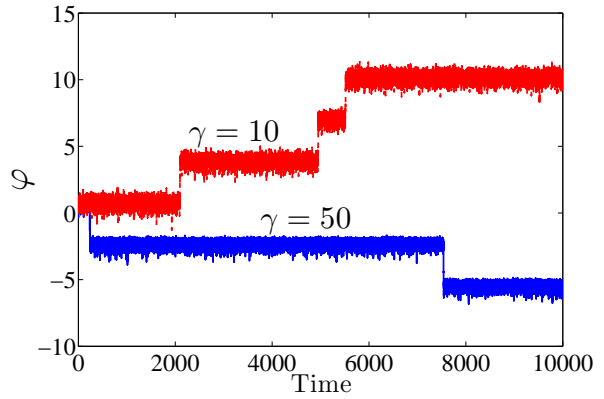

Figure 10: AR system is drift dominated. Left panels ((a), (c), (e), and (g)) show the Ito drift (solid lines) and diffusivity (dashed lines) of the phase reduced system (3.5). Solid vertical lines indicate the ratchet points (where the diffusivity is zero) and the horizontal arrows indicate the sign of the Ito drift at the ratchet points. Right panels ((b), (d), (f), and (h)) show sample phase trajectories of the full system (3.4). For all plots, $c=20, \omega=1$, and unless indicated otherwise $\gamma=50$. (a,b) $K_{A R}=3.5$, (c,d) $K_{A R}=3.961,(\mathrm{e}, \mathrm{f}) K_{A R}=4$, and $(\mathrm{g}, \mathrm{h}) K_{A R}=6.23$ 


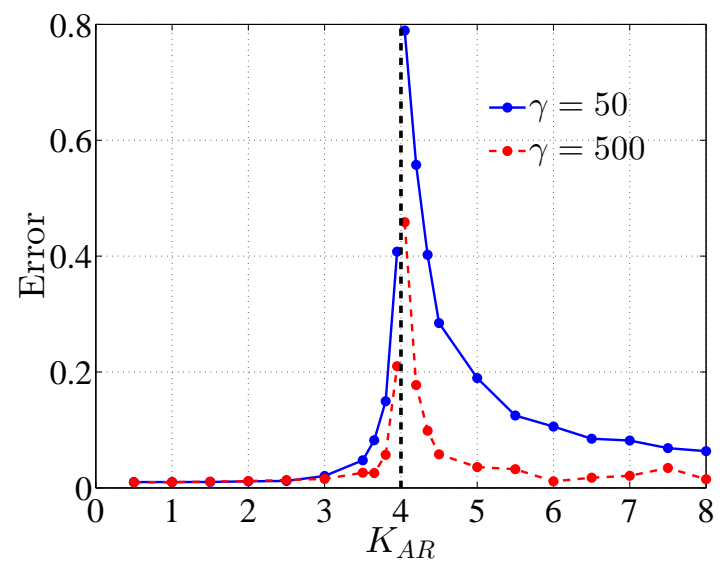

Figure 11: Error in the phase model approximation of the stationary density for the AR system as a function of $K_{A R} . L^{2}$ norm of the difference between the stationary density obtained from solving (3.6) and the stationary density obtained from Monte-Carlo simulations of the full system (3.4) as a function of $K_{A R}$ when $c=20, \omega=1, \gamma=50$ (solid line), and $\gamma=500$ (dashed line). Note that when $K_{A R}>4$, the switching in the AR system becomes rare. To numerically compute the stationary density in this case, we took advantage of the fact that the stationary density is symmetric around $\varphi=\pi / \omega$. Thus, we simulated trajectories of (3.4) with $d t=0.0005$ and $T=1,000$ for 5 different random initial phases in $[0, \pi / \omega)$. These trajectories were then combined and binned to create a histogram for $\varphi \in[0, \pi)$. These binned counts were then repeated for $\varphi \in[\pi / \omega, 2 \pi / \omega)$ (using the symmetry), and then the whole histogram was normalized. For $K_{A R}<4$, we simulated (3.4) with the same time step and $T=5,000$. 
(a)

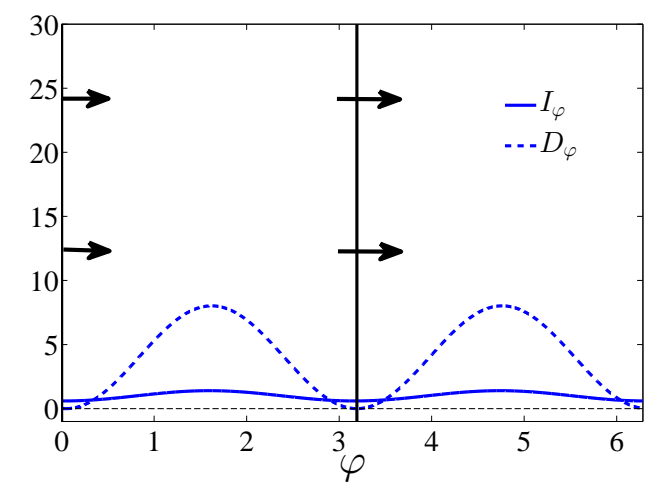

(c)

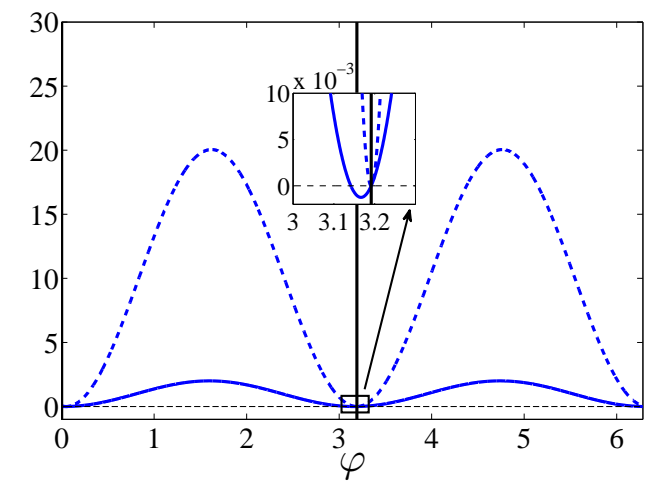

(e)



(b)

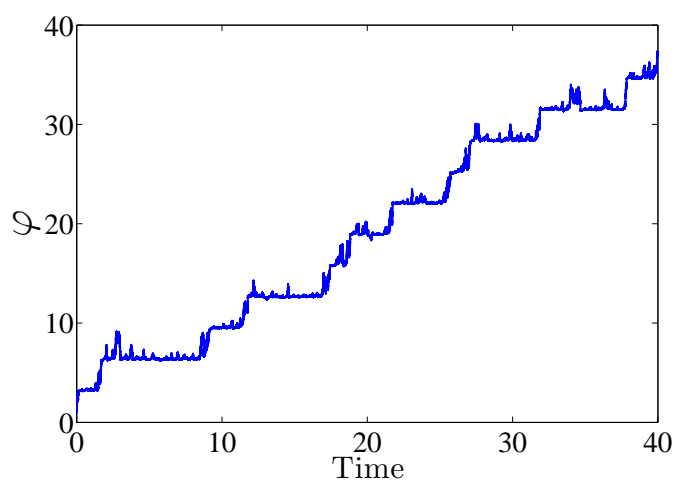

(d)

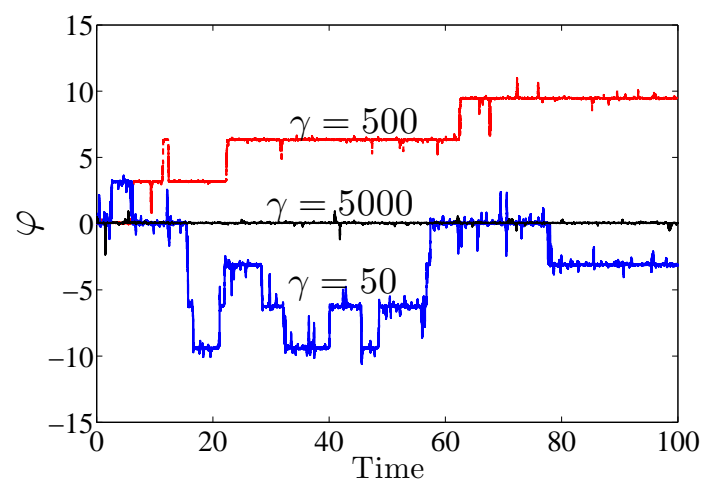

(f)

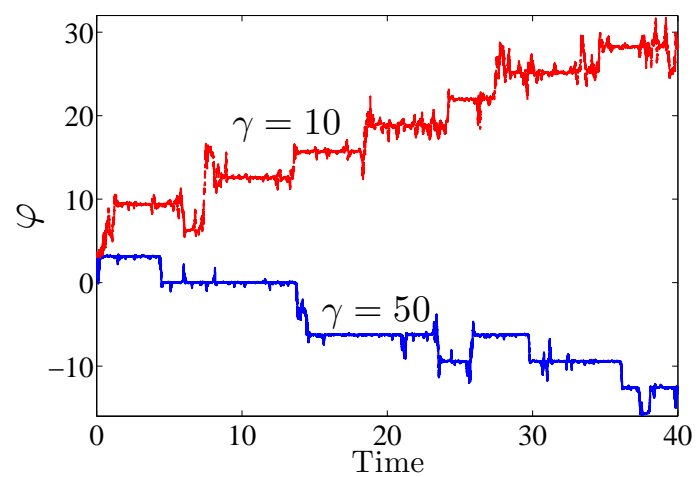

Figure 12: CR system is diffusion dominated. Left panels ((a), (c), and (e)) show the Ito drift (solid lines) and diffusivity (dashed lines) of the phase reduced system (3.5). Solid vertical lines indicate the ratchet points (where the diffusivity is zero) and the horizontal arrows indicate the sign of the Ito drift at the ratchet points. Right panels ((b), (d), and (f)) show sample phase trajectories of the full system (3.4). For all plots, $c=20, \omega=1$, and unless indicated otherwise $\gamma=50$. (a,b) $K_{C R}=4$, (c,d) $K_{C R}=6.32$, and $(\mathrm{e}, \mathrm{f}) K_{C R}=7.5$. 


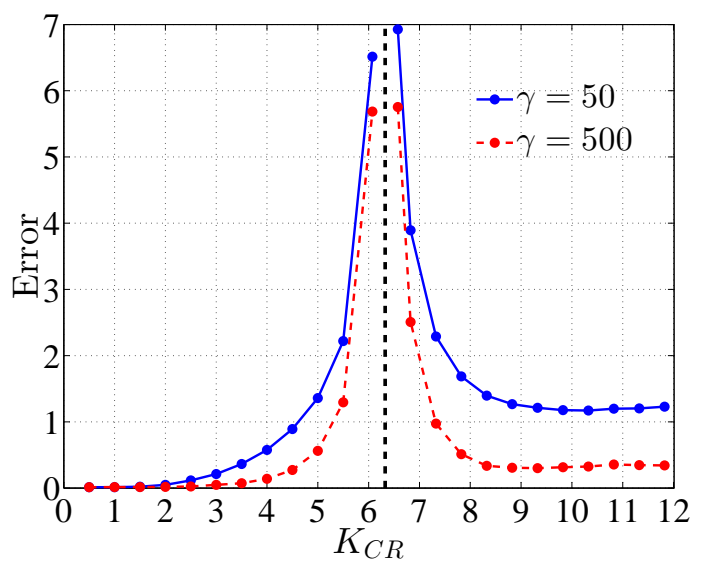

Figure 13: Error in the phase model approximation of the stationary density for the CR system as a function of $K_{C R} . L^{2}$ norm of the difference between the stationary density obtained from solving (3.6) and the stationary density obtained from Monte-Carlo simulations of the full system (3.4) as a function of $K_{C R}$ when $c=20, \omega=1, \gamma=50$ (solid line), and $\gamma=500$ (dashed line). The Monte-Carlo stationary densities were obtained by simulating (3.4) with $d t=0.0005$ and $T=4,000$. 